\title{
Magneto - thermoelectric Effects in Quantum Well in the Presence of Electromagnetic Wave
}

\author{
Nguyen Quang Bau*, Dao Thu Hang, Doan Minh Quang, Nguyen Thi Thanh Nhan \\ Faculty of Physics, VNU University of Science, 334 Nguyen Trai, Hanoi, Vietnam \\ Received 15 March 2017 \\ Revised 16 April 2017; Accepted 20 May 2017
}

\begin{abstract}
We have studied magneto-thermoelectric effects in quantum well in the presence of electromagnetic wave. The analytic expression for Ettingshausen coefficient (EC) in the Quantum Well with parabolic potential (QWPP) in the presence of Electromagnetic wave (EMW) is calculated by using the quantum kinetic equation for electrons. The dependence of EC on the frequency, the amplitude of EMW, the Quantum Well parameters and the temperature are obtained. The results are numerically calculated, plotted, and discussed for GaAs/GaAsAl Quantum Well to clearly show the dependence of EC on above parameters and the results in this case are compared with the case in the bulk semiconductors. We realize that as the temperature increases, the EC decreases. The results show appearance of the Shubnikov-de Haas ( $\mathrm{SdH})$ oscillations when we survey the dependence of EC on the magnetic field.
\end{abstract}

Keywords: Ettingshausen, Quantum well, Electromagnetic wave, parabolic potential, GaAs/GaAsAl.

\section{Introduction}

The magneto-thermoelectric effect has been studied both theoretically and experimentally. In [1, 2], the theory of the Ettingshausen effect in the bulk semiconductors has been also investigated. According to the Hicks and Dresselhaus [3] predicted that "the thermoelectric figure of merit for twodimensional QWs and one-dimensional quantum wires should be substantially enhanced relative to the corresponding bulk materials". In [4], the mechanism for the increase of thermoelectric power of ntype multivalley $\mathrm{PbTe} / \mathrm{Pb} 1-\mathrm{xEuxTe} \mathrm{QWs}$ has been studied theoretically. The theory of thermopower in quantum dots was developed in [5]. The theory of the quantum thermomagnetic effects in sizequantized systems was studied in [6]. The Ettingshausen effect of a two-dimensional electron gas has been investigated theoretically within the framework of the Boltzmann kinetic equation for different mechanisms of electronic scattering taking into account phonon-grag contributions [7]. However, the limitation of the Boltzmann kinetic equation is that it is only used in high temperature conditions and the Ettingshausen effect in the QWPP under the influence of EMW has not been studied. So, in this

\footnotetext{
*Corresponding author. Tel.: 84-913348020.

Email: nguyenquangbau54@gmail.com

https://doi.org/10.25073/2588-1124/vnumap.4071
} 
work, we use the quantum kinetic equation method to calculate the EC in the QWPP under the influence of electromagnetic wave. We see some differences between the results obtained in this case and in the case of the bulk semiconductors. Numerical calculations are carried out with a specific GaAs/GaAsAl Quantum Well. Numerical results and discussion for the GaAs/AlAs cylindrical quantum wire are given in the section 3. And the final section shows remarks and conclusions.

\section{Calculation of ettingshausen coefficient in quantum well in the presence of electromagnetic wave}

In this report, we use quantum kinetic equation method to obtain EC in QWPP in the presence of EMW. We consider a QWPP subjected to a crossed electric field $\vec{E}_{1}=\left(E_{1}, 0,0\right)$, magnetic field $\vec{B}=(0,0, B)$ is perpendicular to the plane of the free electronics .

If the confinement potential is assumed to take the form $V(z)=m \omega_{z}^{2} z^{2} / 2$ then the single-particle wave function and its eigenenergy are given by:

$$
\begin{aligned}
& \psi(\vec{r})=\frac{1}{\sqrt{L_{y}}} \phi_{N}\left(x-x_{0}\right) e^{i k_{y} y} \phi(z) \\
& \varepsilon_{N}\left(k_{x}\right)=\hbar \omega_{c}\left(N+\frac{1}{2}\right)+\left(n+\frac{1}{2}\right) \hbar \omega_{z}-\hbar v k_{y}+\frac{1}{2} m v^{2}
\end{aligned}
$$

Here: $\vec{k}_{y}$ and $L_{y}$ are the wave vector and the normalization length in the y-direction, respectively, $\omega_{z}$ and $\omega_{c}=\frac{e B}{m}$ are the confinement frequency and the cyclotron frequency, respectively. $\mathrm{N}$ is the Landau level index and $\mathrm{n}$ being the subband index, $v=\frac{E_{1}}{B}$ being drift velocity of electron. The Hamiltonian of the electron-acoustic phonon system in QWPP in the second quantization presentation can be written as :

$$
\begin{aligned}
& H=\sum_{N, n, \overrightarrow{k_{y}}} \varepsilon_{N, n}\left(\overrightarrow{k_{y}}-\frac{e}{\hbar c} \vec{A}(t)\right) a_{N, n, \overline{k_{y}}}^{+} a_{N, n, \overrightarrow{k_{y}}}+\sum_{\vec{q}} \hbar \omega_{q} b_{\vec{q}}^{+} b_{\vec{q}}+ \\
& +\sum_{N, n, N^{\prime}, n, \overrightarrow{k_{y}}, \vec{q}} C_{N \cdot N^{\prime}}(\vec{q}) a_{N^{\prime}, n^{\prime}, \overline{k_{y}}+\overrightarrow{q_{y}}}^{+} a_{N, n, \overrightarrow{k_{y}}}\left(b_{\vec{q}}+b_{-\vec{q}}^{+}\right)
\end{aligned}
$$

Where $\vec{A}(t)$ is the vector potential of laser field, ${ }^{\hbar \omega_{q}}$ is the energy of an acoustic phonon with the ware vector $\vec{q}=\left(\vec{q}_{\perp}, q_{z}\right), a_{N, n, \vec{k}_{y}}^{+}$and $a_{N, n, \vec{k}_{y}}\left(b_{\vec{q}}^{+}\right.$and $\left.b_{\vec{q}}\right)$ are the creation and annihilation operators of electron (phonon), respectively.

$$
\left|C_{N, n, N^{\prime}, n^{\prime}}(\vec{q})\right|^{2}=\left|C_{\vec{q}}\right|^{2} \cdot\left|I_{n, n^{\prime}}\left( \pm q_{z}\right)\right|^{2}\left|I_{N, N^{\prime}}(u)\right|^{2}
$$

With: 
$C_{\vec{q}}$ is the electron-phonon interaction constant which depends on the scattering mechanism, $I_{n, n^{\prime}}\left( \pm q_{z}\right)$ is the form factor of electron, given by:

$$
\begin{aligned}
& I_{n, n^{\prime}}\left( \pm q_{z}\right)=\frac{1}{\sqrt{\pi} l_{z}} \frac{1}{\sqrt{2^{n} n ! 2^{n^{\prime}} n ! !}} \int_{-\infty}^{+\infty} e^{ \pm i q_{z} z} e^{-z^{2} / l_{z}^{2}} H_{n}\left(\frac{z}{l_{z}}\right) H_{n^{\prime}}\left(\frac{z}{l_{z}}\right) d z \\
& \left|I_{N, N^{\prime}}(u)\right|^{2}=\frac{N^{\prime} !}{N !} e^{-u} u^{N^{\prime}-N}\left[L_{N}^{N^{\prime}-N}(u)\right]^{2}
\end{aligned}
$$

Where

$$
\begin{aligned}
& L_{M}^{N}(x) \text { is the associated Laguerre polynomial. } \\
& u=l_{B}^{2} q_{\perp}^{2} / 2, l_{B}=\sqrt{\hbar /\left(m \omega_{c}\right)}, q_{\perp}^{2}=q_{x}^{2}+q_{y}^{2} .
\end{aligned}
$$

When a high-frequency EMW is applied to the system in the $\mathrm{z}$ direction with electric field vector $\vec{E}=\overrightarrow{E_{0}} \sin \Omega t$ (where $\vec{E}_{0}$ and $\Omega$ are the amplitude and the frequency of the EMW), the quantum kinetic equation of average number of electron $f_{N, n, \overrightarrow{k_{y}}}=\left\langle a_{N, n, \overrightarrow{k_{y}}}^{+} a_{N, n, \overrightarrow{k_{y}}}\right\rangle_{t}$ is:

$$
i \hbar \frac{\partial\left\langle a_{N, n, \overline{k_{y}}}^{+} a_{N, n, \overline{k_{y}}}\right\rangle_{t}}{\partial t}=\left\langle\left[a_{N, n, \overline{k_{y}}}^{+} a_{N, n, \overline{k_{y}}}, H\right]\right\rangle_{t}
$$

By replacing Eq.(3) on Eq.(6) we get the quantum kinetic equation:

$$
\begin{aligned}
& \frac{\partial f_{N, n, \overrightarrow{k_{y}}}}{\partial t}+\left(\frac{e \overrightarrow{E_{1}}}{\hbar}+\frac{\omega_{c}}{\hbar}\left[\overrightarrow{k_{x}}, \vec{h}\right]\right) \frac{\partial f_{N, n, \overrightarrow{k_{y}}}}{\partial \overrightarrow{k_{y}}}=\frac{2 \pi}{\hbar} \sum_{N^{\prime}, n^{\prime}, \vec{q}}\left|C_{N, n, N^{\prime}, n^{\prime}}(\vec{q})\right|^{2} \\
& \times \sum_{l=-\infty}^{\infty} J_{l}^{2}\left(\frac{\lambda}{\Omega}\right)\left(2 N_{\vec{q}}+1\right)\left\{\left(f_{N^{\prime}, n^{\prime}, \overrightarrow{k_{y}}+\overrightarrow{q_{y}}}\left(N_{\vec{q}}+1\right)-f_{N, n, \overrightarrow{k_{y}}} N_{\vec{q}}\right)\right. \\
& \times \delta\left(\varepsilon_{N^{\prime}, n^{\prime}, \overline{k_{y}}+\overrightarrow{q_{y}}}-\varepsilon_{N, n, \overline{k_{y}}}-\hbar \omega_{\vec{q}}-l \hbar \Omega\right)+\left(f_{N^{\prime}, n^{\prime}, \overline{k_{y}}}-\bar{q}_{y} N_{\vec{q}}-f_{N, n, \overline{y_{y}}}\right. \\
& \left.\left.\times\left(N_{\vec{q}}+1\right)\right) \delta\left(\varepsilon_{N^{\prime}, n^{\prime}, \overline{k_{y}}}-\bar{q}_{y}-\varepsilon_{N, n, \overline{k_{y}}}+\hbar \omega_{\vec{q}}-l \hbar \Omega\right)\right\}
\end{aligned}
$$

Here $\vec{h}=\frac{\vec{H}}{H}$ is unit vector in the direction of magnetic field. For simplicity, we limit the problem to case of $l=-1,0,1$. Now, we mutiply both sides of the Eq.(7) by $(e / m) \overrightarrow{k_{y}} \delta\left(\varepsilon-\varepsilon_{N, n, k_{y}}\right)$, carry out the summation over $\mathrm{N}$ and $\vec{k}_{y}$ and then notice that $J_{0}^{2}\left(\frac{\lambda}{\Omega}\right) \approx 1-\left(\frac{\lambda}{\Omega}\right)^{2} / 2$, we get following equation: 
$\frac{\vec{G}(\varepsilon)}{\tau(\varepsilon)}+\omega_{c}[\vec{h}, \vec{G}(\varepsilon)]=\vec{P}(\varepsilon)+\vec{M}(\varepsilon)$,

Where:

$$
\vec{P}(\varepsilon)=-\frac{e}{m} \sum_{N, n, \vec{k}_{y}} \overrightarrow{k_{y}}\left(\vec{F}, \frac{\partial f_{N, n, \overrightarrow{k_{y}}}}{\partial \overrightarrow{k_{y}}}\right) \delta\left(\varepsilon-\varepsilon_{N, n, \vec{k}_{y}}\right),
$$

with

$$
\vec{F}=e \overrightarrow{E_{1}}-\nabla \varepsilon_{F}-\frac{\varepsilon-\varepsilon_{F}}{T} \nabla T .
$$

$\tau$ is the momentum relaxation time.

$$
\begin{aligned}
& \vec{M}(\varepsilon)=-\frac{4 \pi e}{\hbar m} \sum_{N^{\prime}, n^{\prime}, \vec{q}} \sum_{N, n, \overrightarrow{k_{y}}}\left|C_{N, n, N^{\prime}, n^{\prime}}(q)\right|^{2} N_{\vec{q}} \vec{k}_{y}\left(f_{N^{\prime}, n^{\prime}, \overrightarrow{k_{y}}+\overrightarrow{q_{y}}}-f_{N, n, \overrightarrow{k_{y}}}\right) \\
& \times\left\{\delta\left(\varepsilon_{N^{\prime}, n^{\prime}, \overrightarrow{k_{y}}}+\overrightarrow{q_{y}}-\varepsilon_{N, n, \overrightarrow{k_{y}}}\right)\left(1-\frac{\lambda^{2}}{2 \Omega^{2}}\right)+\frac{\lambda^{2}}{4 \Omega^{2}} \delta\left(\varepsilon_{N^{\prime}, n^{\prime}, \overrightarrow{k_{y}}+\overrightarrow{q_{y}}}-\varepsilon_{N, n, \overrightarrow{k_{y}}}+\hbar \Omega\right)\right. \\
& \left.+\frac{\lambda^{2}}{4 \Omega^{2}} \delta\left(\varepsilon_{N^{\prime}, n^{\prime}, \overrightarrow{k_{y}}}+\overrightarrow{q_{y}}-\varepsilon_{N, n, \overrightarrow{k_{y}}}+\hbar \Omega\right)\right\} \delta\left(\varepsilon-\varepsilon_{N, n, \overrightarrow{k_{y}}}\right) \\
& \vec{G}(\varepsilon)=\sum_{N, n, \bar{k}_{y}} \frac{e}{m} \overrightarrow{k_{y}} f_{N, n, \overline{k_{y}}} \delta\left(\varepsilon-\varepsilon_{N, n, \bar{k}_{y}}\right)
\end{aligned}
$$

The current density $\overrightarrow{\boldsymbol{J}}$ and thermal flux density $\vec{q}_{e}$ given by:

$$
\begin{aligned}
& \vec{J}=\int_{0}^{\infty} \vec{G}(\varepsilon) d \varepsilon \\
& \overrightarrow{q_{e}}=\frac{1}{e} \int_{0}^{\infty}\left(\varepsilon-\varepsilon_{F}\right) \vec{G}(\varepsilon) d \varepsilon
\end{aligned}
$$

From the current density and thermal flux density formula, we obtain the EC:

$$
P=\frac{1}{B} \frac{\sigma_{x x} \gamma_{x y}-\sigma_{x y} \gamma_{x x}}{\sigma_{x x}\left[\beta_{x x} \gamma_{x x}-\sigma_{x x}\left(\xi_{x x}-K_{L}\right)\right]}
$$

with: 


$$
\begin{gathered}
\sigma_{i m}=a \frac{\tau\left(\varepsilon_{F}\right)}{1+\omega_{c}^{2} \tau^{2}\left(\varepsilon_{F}\right)}\left[\delta_{\mathrm{ij}}-\omega_{c} \tau\left(\varepsilon_{F}\right) \varepsilon_{\mathrm{ijk}} h_{k}+\omega_{c}^{2} \tau^{2}\left(\varepsilon_{F}\right) h_{i} h_{j}\right] \delta_{j m}+\frac{e}{m} \times \\
\times\left[\frac{\tau\left(B_{1}-\mathrm{eE}_{1} \overline{\Delta x}\right)}{1+\omega_{c}^{2} \tau^{2}\left(B_{1}-\mathrm{eE}_{1} \overline{\Delta x}\right)}\right]^{2}\left[\delta_{\mathrm{ij}}-\omega_{c} \tau\left(B_{1}-\mathrm{eE}_{1} \overline{\Delta x}\right) \varepsilon_{\mathrm{ijk}} h_{k}+\omega_{c}^{2} \tau^{2}\left(B_{1}-\mathrm{e} \mathrm{E}_{1} \overline{\Delta x}\right) h_{i} h_{j}\right] \delta_{j l} \times\left[\delta_{l m}-\omega_{c} \tau \times\right. \\
\left.\times\left(B_{1}-\mathrm{eE}_{1} \overline{\Delta x}\right) \varepsilon_{l m p} h_{p}+\omega_{c}^{2} \tau^{2}\left(B_{1}-\mathrm{eE}_{1} \overline{\Delta x}\right) h_{l} h_{m}\right]+\frac{e}{m}\left[\frac{\tau\left(B_{1}-\mathrm{eE}_{1} \overline{\Delta x}-\hbar \Omega\right)}{1+\omega_{c}^{2} \tau^{2}\left(B_{1}-\mathrm{eE}_{1} \overline{\Delta x}-\hbar \Omega\right)}\right]^{2}\left[\varepsilon_{\mathrm{ijk}} h_{k}+\omega_{c}^{2} \tau^{2}\right. \\
\left.\left.\times\left(B_{1}-\mathrm{eE}_{1} \overline{\Delta x}-\hbar \Omega\right) h_{i} h_{j}\right] \delta_{j l}\left[\delta_{l m}-\omega_{c} \tau\left(B_{1}-\mathrm{eE}_{1} \overline{\Delta x}-\hbar \Omega\right) \varepsilon_{l m p} h_{p}+\omega_{c}^{2} \tau^{2}\left(B_{1}-\mathrm{eE} \bar{\Delta}_{1} \overline{\Delta x}-\hbar \Omega\right) h_{l} h_{m}\right]\right] \\
+\frac{e}{m}\left[\frac{\tau\left(B_{1}-\mathrm{eE}_{1} \overline{\Delta x}+\hbar \Omega\right)}{1+\omega_{c}^{2} \tau^{2}\left(B_{1}-\mathrm{e} \mathrm{E}_{1} \overline{\Delta x}+\hbar \Omega\right)}\right]^{2}\left[\delta_{\mathrm{ij}}-\omega_{c} \tau\left(B_{1}-\mathrm{e} \mathrm{E}_{1} \overline{\Delta x}+\hbar \Omega\right) \varepsilon_{\mathrm{ijk}} h_{k}+\omega_{c}^{2} \tau^{2}\left(B_{1}-\mathrm{eE} \overline{\Delta x}+\hbar \Omega\right) h_{i} h_{j}\right] \\
\left.\left.\delta_{j l}\left[\delta_{l m}-\omega_{c} \tau\left(B_{1}-\mathrm{eE}_{1} \overline{\Delta x}+\hbar \Omega\right) \times \varepsilon_{l m p} h_{p}+\omega_{c}^{2} \tau^{2}\left(B_{1}-\mathrm{eE}_{1} \overline{\Delta x}+\hbar \Omega\right) h_{l} h_{m}\right]\right]\right]
\end{gathered}
$$$$
\beta_{i m}=-\left\{\frac { e ( B _ { 1 } - \mathrm { eE } _ { 1 } \overline { \Delta x } - \varepsilon _ { F } ) } { T m } [ \frac { \tau ( B _ { 1 } - \mathrm { eE } _ { 1 } \overline { \Delta x } ) } { 1 + \omega _ { c } ^ { 2 } \tau ^ { 2 } ( B _ { 1 } - \mathrm { eE } _ { 1 } \overline { \Delta x } ) } ] ^ { 2 } \left[\delta_{\mathrm{ij}}-\omega_{c} \tau\left(B_{1}-\mathrm{eE}_{1} \overline{\Delta x}\right) \varepsilon_{\mathrm{ij} k} h_{k}+\omega_{c}^{2} \tau^{2}\left(B_{1}-\mathrm{eE}_{1} \overline{\Delta x}\right)\right.\right.
$$$$
\left.\times h_{i} h_{j}\right] \delta_{j l}\left[\delta_{l m}-\omega_{c} \tau\left(B_{1}-\mathrm{eE}_{1} \overline{\Delta x}\right) \varepsilon_{l m p} h_{p}+\omega_{c}^{2} \tau^{2}\left(B_{1}-\mathrm{eE}_{1} \overline{\Delta x}\right) h_{l} h_{m}\right]+\frac{e\left(B_{1}-\mathrm{eE}_{1} \overline{\Delta x}-\hbar \Omega-\varepsilon_{F}\right)}{T m} \times
$$$$
\times\left[\frac{\tau\left(B_{1}-\mathrm{eE}_{1} \overline{\Delta x}-\hbar \Omega\right)}{1+\omega_{c}^{2} \tau^{2}\left(B_{1}-\mathrm{eE}_{1} \overline{\Delta x}-\hbar \Omega\right)}\right]^{2} \times\left[\delta_{\mathrm{ij}}-\omega_{c} \tau\left(B_{1}-\mathrm{eE}_{1} \overline{\Delta x}-\hbar \Omega\right) \varepsilon_{\mathrm{ij} k} h_{k}+\omega_{c}^{2} \tau^{2}\left(B_{1}-\mathrm{eE}_{1} \overline{\Delta x}-\hbar \Omega\right) h_{i} h_{j}\right] \delta_{j l}
$$$$
\left.\times\left[\delta_{l m}-\omega_{c} \tau\left(B_{1}-\mathrm{eE}_{1} \overline{\Delta x}-\hbar \Omega\right) \varepsilon_{l m p} h_{p}+\omega_{c}^{2} \tau^{2}\left(B_{1}-\mathrm{eE}_{1} \overline{\Delta x}-\hbar \Omega\right) h_{l} h_{m}\right]\right]+\frac{e\left(B_{1}-\mathrm{eE}_{1} \overline{\Delta x}+\hbar \Omega-\varepsilon_{F}\right)}{T m} \times
$$$$
\times\left[\frac{\tau\left(B_{1}-\mathrm{eE}_{1} \overline{\Delta x}+\hbar \Omega\right)}{1+\omega_{c}^{2} \tau^{2}\left(B_{1}-\mathrm{eE}_{1} \overline{\Delta x}+\hbar \Omega\right)}\right]^{2}\left[\delta_{\mathrm{ij}}-\omega_{c} \tau\left(B_{1}-\mathrm{eE}_{1} \overline{\Delta x}+\hbar \Omega\right) \varepsilon_{\mathrm{ijk}} h_{k}+\omega_{c}^{2} \tau^{2}\left(B_{1}-\mathrm{eE}_{1} \overline{\Delta x}+\hbar \Omega\right) h_{i} h_{j}\right] \delta_{j l}
$$$$
\left.\left.\left.\times\left[\delta_{l m}-\omega_{c} \tau\left(B_{1}-\mathrm{eE}_{1} \overline{\Delta x}+\hbar \Omega\right) \times \varepsilon_{l m p} h_{p}+\omega_{c}^{2} \tau^{2}\left(B_{1}-\mathrm{eE}_{1} \overline{\Delta x}+\hbar \Omega\right) h_{l} h_{m}\right]\right]\right]\right\}
$$ 


$$
\begin{aligned}
& \gamma_{i m}=\frac{\left(B_{1}-\mathrm{eE}_{1} \overline{\Delta x}-\varepsilon_{F}\right)}{m}\left[\frac{\tau\left(B_{1}-\mathrm{eE}_{1} \overline{\Delta x}\right)}{1+\omega_{c}^{2} \tau^{2}\left(B_{1}-\mathrm{eE}_{1} \overline{\Delta x}\right)}\right]^{2}\left[\delta_{\mathrm{ij}}-\omega_{c} \tau\left(B_{1}-\mathrm{eE}_{1} \overline{\Delta x}\right) \varepsilon_{\mathrm{ij} k} h_{k}+\omega_{c}^{2} \tau^{2}\left(B_{1}-\mathrm{eE}_{1} \overline{\Delta x}\right) \times\right. \\
& \left.\times h_{i} h_{j}\right] \delta_{j l}\left[\delta_{l m}-\omega_{c} \tau\left(B_{1}-\mathrm{eE}_{1} \overline{\Delta x}\right) \varepsilon_{l m p} h_{p}+\omega_{c}^{2} \tau^{2}\left(B_{1}-\mathrm{eE}_{1} \overline{\Delta x}\right) h_{l} h_{m}\right]+\frac{\left(B_{1}-\mathrm{eE}_{1} \overline{\Delta x}-\hbar \Omega-\varepsilon_{F}\right)}{m} \times \\
& \times\left[\frac{\tau\left(B_{1}-\mathrm{eE}_{1} \overline{\Delta x}-\hbar \Omega\right)}{1+\omega_{c}^{2} \tau^{2}\left(B_{1}-\mathrm{eE}_{1} \overline{\Delta x}-\hbar \Omega\right)}\right]^{2} \times\left[\delta_{\mathrm{ij}}-\omega_{c} \tau\left(B_{1}-\mathrm{eE}_{1} \overline{\Delta x}-\hbar \Omega\right) \varepsilon_{\mathrm{ijk}} h_{k}+\omega_{c}^{2} \tau^{2}\left(B_{1}-\mathrm{eE}_{1} \overline{\Delta x}-\hbar \Omega\right) h_{i} h_{j}\right] \delta_{j l} \\
& \left.\times\left[\delta_{l m}-\omega_{c} \tau\left(B_{1}-\mathrm{eE}_{1} \overline{\Delta x}-\hbar \Omega\right) \varepsilon_{l m p} h_{p}+\omega_{c}^{2} \tau^{2}\left(B_{1}-\mathrm{eE}_{1} \overline{\Delta x}-\hbar \Omega\right) h_{l} h_{m}\right]\right]+\frac{\left(B_{1}-\mathrm{eE}_{1} \overline{\Delta x}+\hbar \Omega-\varepsilon_{F}\right)}{m} \times \\
& \times\left[\frac{\tau\left(B_{1}-\mathrm{eE}_{1} \overline{\Delta x}+\hbar \Omega\right)}{1+\omega_{c}^{2} \tau^{2}\left(B_{1}-\mathrm{eE}_{1} \overline{\Delta x}+\hbar \Omega\right)}\right]^{2}\left[\delta_{\mathrm{ij}}-\omega_{c} \tau\left(B_{1}-\mathrm{eE}_{1} \overline{\Delta x}+\hbar \Omega\right) \varepsilon_{\mathrm{ijk}} h_{k}+\omega_{c}^{2} \tau^{2}\left(B_{1}-\mathrm{eE}_{1} \overline{\Delta x}+\hbar \Omega\right) h_{i} h_{j}\right] \delta_{j l} \\
& \left.\left.\times\left[\delta_{l m}-\omega_{c} \tau\left(B_{1}-\mathrm{eE}_{1} \overline{\Delta x}+\hbar \Omega\right) \times \varepsilon_{l m p} h_{p}+\omega_{c}^{2} \tau^{2}\left(B_{1}-\mathrm{eE}_{1} \overline{\Delta x}+\hbar \Omega\right) h_{l} h_{m}\right]\right]\right] \\
& \beta_{i m}=-\left\{\frac { ( B _ { 1 } - \mathrm { eE } _ { 1 } \overline { \Delta x } - \varepsilon _ { F } ) ^ { 2 } } { T m } [ \frac { \tau ( B _ { 1 } - \mathrm { eE } _ { 1 } \overline { \Delta x } ) } { 1 + \omega _ { c } ^ { 2 } \tau ^ { 2 } ( B _ { 1 } - \mathrm { eE } _ { 1 } \overline { \Delta x } ) } ] ^ { 2 } \left[\delta_{\mathrm{ij}}-\omega_{c} \tau\left(B_{1}-\mathrm{eE}_{1} \overline{\Delta x}\right) \varepsilon_{\mathrm{ijk}} h_{k}+\omega_{c}^{2} \tau^{2}\left(B_{1}-\mathrm{eE}_{1} \overline{\Delta x}\right)\right.\right. \\
& \left.\times h_{i} h_{j}\right] \delta_{j l}\left[\delta_{l m}-\omega_{c} \tau\left(B_{1}-\mathrm{eE}_{1} \overline{\Delta x}\right) \varepsilon_{l m p} h_{p}+\omega_{c}^{2} \tau^{2}\left(B_{1}-\mathrm{eE}_{1} \overline{\Delta x}\right) h_{l} h_{m}\right]+\frac{\left(B_{1}-\mathrm{eE}_{1} \overline{\Delta x}-\hbar \Omega-\varepsilon_{F}\right)^{2}}{T m} \times \\
& \times\left[\frac{\tau\left(B_{1}-\mathrm{eE}_{1} \overline{\Delta x}-\hbar \Omega\right)}{1+\omega_{c}^{2} \tau^{2}\left(B_{1}-\mathrm{eE}_{1} \overline{\Delta x}-\hbar \Omega\right)}\right]^{2} \times\left[\delta_{\mathrm{ij}}-\omega_{c} \tau\left(B_{1}-\mathrm{eE}_{1} \overline{\Delta x}-\hbar \Omega\right) \varepsilon_{\mathrm{ijk}} h_{k}+\omega_{c}^{2} \tau^{2}\left(B_{1}-\mathrm{eE}_{1} \overline{\Delta x}-\hbar \Omega\right) h_{i} h_{j}\right] \delta_{j l} \\
& \left.\times\left[\delta_{l m}-\omega_{c} \tau\left(B_{1}-\mathrm{eE}_{1} \overline{\Delta x}-\hbar \Omega\right) \varepsilon_{l m p} h_{p}+\omega_{c}^{2} \tau^{2}\left(B_{1}-\mathrm{eE}_{1} \overline{\Delta x}-\hbar \Omega\right) h_{l} h_{m}\right]\right]+\frac{\left(B_{1}-\mathrm{eE}_{1} \overline{\Delta x}+\hbar \Omega-\varepsilon_{F}\right)^{2}}{T m} \times \\
& \times\left[\frac{\tau\left(B_{1}-\mathrm{eE}_{1} \overline{\Delta x}+\hbar \Omega\right)}{1+\omega_{c}^{2} \tau^{2}\left(B_{1}-\mathrm{eE}_{1} \overline{\Delta x}+\hbar \Omega\right)}\right]^{2}\left[\delta_{\mathrm{ij}}-\omega_{c} \tau\left(B_{1}-\mathrm{eE}_{1} \overline{\Delta x}+\hbar \Omega\right) \varepsilon_{\mathrm{ij} k} h_{k}+\omega_{c}^{2} \tau^{2}\left(B_{1}-\mathrm{eE}_{1} \overline{\Delta x}+\hbar \Omega\right) h_{i} h_{j}\right] \delta_{j l} \\
& \left.\left.\left.\times\left[\delta_{l m}-\omega_{c} \tau\left(B_{1}-\mathrm{eE}_{1} \overline{\Delta x}+\hbar \Omega\right) \times \varepsilon_{l m p} h_{p}+\omega_{c}^{2} \tau^{2}\left(B_{1}-\mathrm{eE}_{1} \overline{\Delta x}+\hbar \Omega\right) h_{l} h_{m}\right]\right]\right]\right\}
\end{aligned}
$$

Here: $B_{1}=\left(N^{\prime}-N\right) \hbar \omega_{c}-\left(n^{\prime}-n\right) \hbar \omega_{z}$. The appearance of the parameter $\overline{\Delta x}$ is due to the replacement of $e B \overline{\Delta x} / \hbar$, where $\overline{\Delta x}$ is a constant of the order of $l_{B}$ [8], $\varepsilon_{F}$ are the Fermi level. From the analytic expression for EC we see that: EC dependence on external fields (i.e. electrical field intensity $\overrightarrow{E_{1}}$, the cyclotron $\omega_{c}$ ), including EMW (i.e. frequency $\Omega$ and amplitude $\overrightarrow{E_{0}}$ of EMW), temperature and special parameters for QWPP (i.e. the confinement frequencies $\omega_{z}$ ), and these 
dependences is more complicated than those in the bulk semiconductors. This result is due to the difference in structure, wave function and energy spectrum of QWPP in comparison with the bulk semiconductors. Moreover, we see that the analytic expression for EC in the QWPP is absolutely different from that in bulk semiconductors. In the next section, we will give a deeper insight into this dependence by carrying out a numerical evaluation.

\section{Numerical results and discussion}

In this section, we present detailed numerical calculations of the EC in a QWPP subjected to uniform crossed magnetic and electric fields in the presence of an EMW. For the numerical evaluation, we consider the model of a QWPP GaAs/AlGaAs with the following parameters:

$\varepsilon_{F}=50 \mathrm{meV} ; \xi=13,5 \mathrm{eV} ; \eta=3.10^{16} \mathrm{~cm}^{-3} ; B=5 T ; \omega_{z}=9,1.10^{14} \mathrm{~s}^{-1} ; \rho=5,32 \mathrm{~g} \cdot \mathrm{cm}^{-3} ; v=5378 \mathrm{~m}^{-1}$

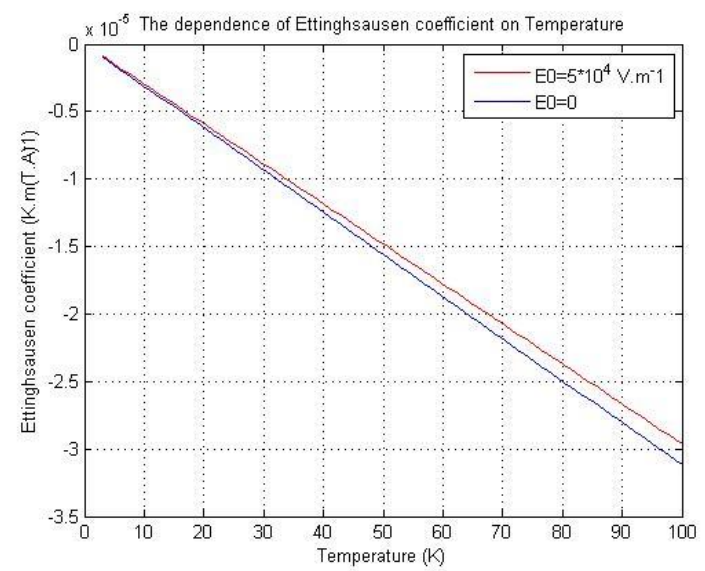

Fig.(1) describes the dependence of EC on temperature with $\Omega=10^{10} \mathrm{~Hz}$,

$$
E_{0}=5.10^{4} \mathrm{~V} / \mathrm{m}, E_{1}=10^{5} \mathrm{~V} / \mathrm{m}, B=5 T .
$$

In the Fig (1): The dependence of the EC in QWPP on temperature is vaguely nonlinear (nearly linear). The EC decreases as the temperature increases. This is consistent with the experimental result obtained in the bulk semiconductors case [1]. However, in the bulk semiconductors, EC has positive value, whereas the EC in QWPP on temperature has negative value. This result is due to the difference in structure, wave function and energy spectrum of QWPP in comparison with the bulk semiconductors. Also, the presence of electromagnetic waves influence on the EC weakly, the EC value is the same in the domain of low temperature and have different values in the region with higher temperatures.

Figure (2) shows the dependence of the EC on the magnetic field. We can see clearly the appearance of oscillations and oscillations are controlled by the ratio of the Fermi energy and energy of cyclotron. The mechanism of the oscillations can be easily explained as follows. At low temperature and strong magnetic field, the free electrons in metals, semiconductors will move as simple harmonic oscillator. When the magnetic field changes, the cycle of the oscillations also changes. The energy levels of electrons are separated into Landau levels, with each level Landau, cyclotron energy and the electron state linearly increase with the magnetic field. When the energy 
level of the Landau levels excesses the value of Fermi level, the electron can move up freely and move in the line, which makes the EC oscillate circulating with magnetic field. The presence of electromagnetic waves influence on the EC weakly. From fig (2) we see that the value of the EC is the same in the domain of weak magnetic field and there is not much different value in the domain of high magnetic field.

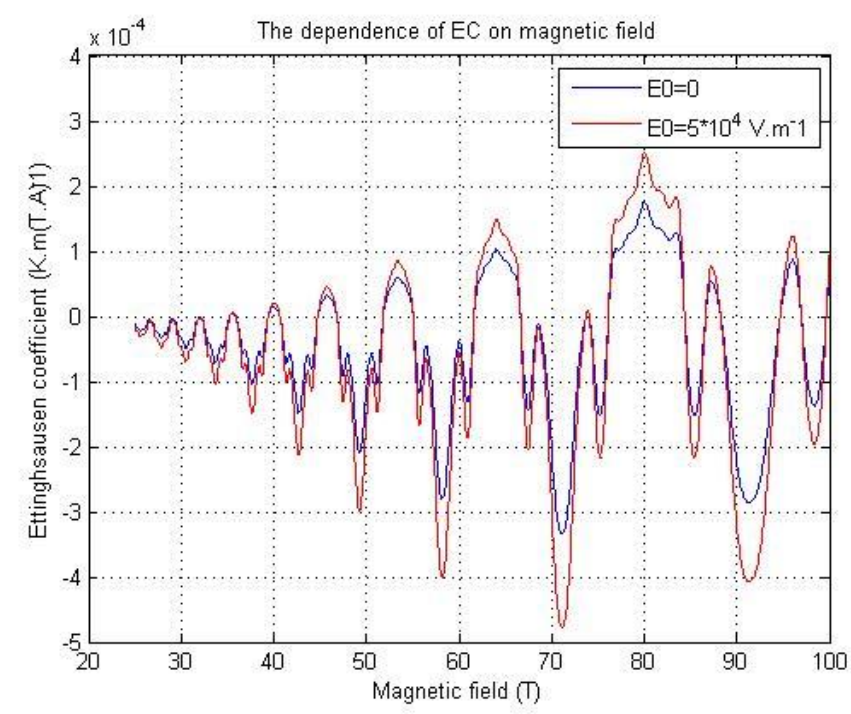

Fig.(2) describes the dependence of EC on magnetic field with $\Omega=10^{10} \mathrm{~Hz}, E_{0}=5.10^{4} \mathrm{~V} / \mathrm{m}, E_{1}=5.10^{5} \mathrm{~V} / \mathrm{m}$.

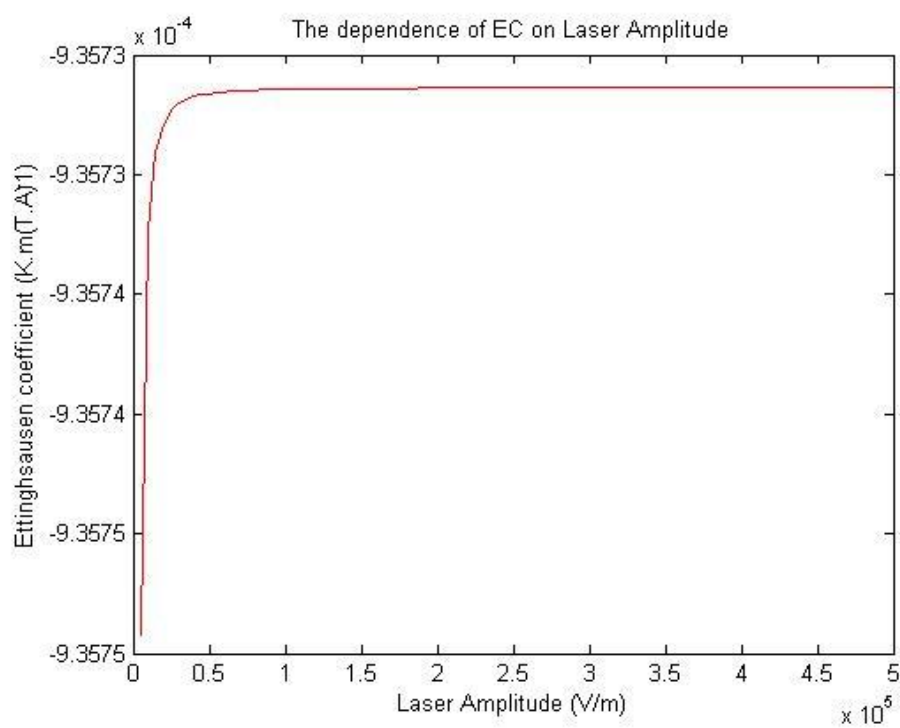

Fig.(3) describes the dependence of EC on laser amplitude with

$$
E_{1}=10^{5} \mathrm{~V} / \mathrm{m}, \mathrm{B}=5 \mathrm{~T}, \Omega=10^{12} \mathrm{~Hz} .
$$


In Figure 3, we investigated the dependence of the EC on amplitude of electromagnetic waves. Basing on the graph, we see that when the amplitude of electromagnetic waves increases, the EC is almost unchanged.

\section{Conclusion}

In this paper, we analytically investigated EC in the Quantum Well in the presence of the EMW with parabolic potential. The electron-phonon interaction is taken into account at low temperatures. We give out the analytical expression of EC in the Quantum Well. Estimating numerical values and graph for a GaAs/GaAsAl Quantum Well to see clearly dependence of the EC on the amplitude of EMW, magnetic field and temperature.

The results showed that the EC decreases linearly with temperature and the EC has a negative value. When surveying the EC dependence on EMW amplitude, we see that the amplitude of electromagnetic wave less impact the EC. In addition, we see the appearance of SdH oscillations when the survey EC dependence on the magnetic field.

\section{Acknowledgments}

This work was completed with financial support from the National Foundation for Science and Technology Development of Vietnam (Nafosted 103.01-2015.22) and Vietnam International Education Development (Project 911).

\section{References}

[1] Paranjape. B.V and Levinger.J.S, Theory of the Ettingshausen effect in semiconductors, Phys. Rev. 120 (1960 ) 437

[2] Malevich. V. L. and Epshtein .E.M, Photostimulated odd magnetoresistance of semiconductors, Sov. Phys. Solid State (Fiz. Tverd. Tela) 18 (1976) 1286

[3] Hicks .L .D and Dresselhaus .M .S, Effect of quantum-well structures on the thermoelectric figure of merit, Phys. Rev. B 47 (1993) 12727

[4] Koga .T, Harman .T .C, Kronin .S .B and Dresselhaus .M .S,Mechanism of the enhanced thermoelectric power in (111)-orientedn-type PbTe/Pb1-xEuxTe multiple quantum wells Phys. Rev. B 60 (1999) 14286

[5] Beenakker .C.W and Staring .A .A,Theory of the thermopower of a quantum dot, Phys. Rev. B 46 (1992) 9667

[6] Sinyavskii .E .P and Khamidullin .R .A, Low-Dimensional Systems, Semiconductors, 36 (2002) 924

[7] G. M. Shmelev, A. V. Yudina, I. I. Maglevanny, and A. S. Bulygin, Electric-Field-Induced Ettingshausen Effect in a Superlattice, Phys.Status Solidi b, 219 (2000) 115.

[8] N. Q. Bau and B. D. Hoi, Investigation of the Hall effect in rectangular quantum wells with a perpendicular magnetic fields in the presence of a high-frequency electromagnetic wave, International Journal of Modern Physics B, 3 (2014) p.1450001-1 